\title{
Intelligentes Wissensmanagement als Game Changer
}

Im Jahr 2020 veränderte die COVID-19-Pandemie weltweit das gesellschaftliche und berufliche Leben massiv. Kontaktbeschränkungen, Abstandsverordnungen, Versammlungsverbote und Homeoffice hatten und haben auf der ganzen Welt nachhaltige Auswirkungen auf alle Unternehmen.

Die digitale Transformation von Geschäftsmodellen (Stichwort: Business Process Transformation) erfuhr einen regelrechten Boost und auch mobiles Arbeiten rückte enorm in den Vordergrund. Dem IT-Analysten Gartner zufolge wird sich dieser Anstieg auch nach dem hoffentlich baldigen Ende der Pandemie noch weiter fortsetzen. So prognostiziert das Marktforschungsunternehmen ein Wachstum des sogenannten Remote Working auf $48 \%$ bis 2030 [1].

Damit ändern sich auch die Anforderungen an Unternehmen, Mitarbeiter und an die eingesetzten Werkzeuge. Um Wissensverlusten vorzubeugen, Abstimmmungsprozesse zu optimieren und den Informationsfluss aufrechtzuerhalten, steigt der Bedarf an innovativen Wissensmanagementlösungen wie „Insight Engines“. Diese nutzen Entwicklungen aus dem Bereich der künstlichen Intelligenz (KI) zur Informationsaufbereitung.

Wieviel KI steckt in einer Insight Engine und welche Vorteile bringt dies für Unternehmen?

Bereits vor Ausbruch der Pandemie verbrachten Mitarbeiter oft unzählige Stunden mit dem Suchen nach Informationen, anstatt sich auf ihre Kernkompetenzen zu konzentrieren. Einer Studie des US-amerikanischen Analystenhauses IDC zufolge vergeuden Wissensarbeiter damit jede Woche $50 \%$ ihrer Arbeitszeit. Demnach verbringen sie $30 \%$ dieser Zeit mit der Suche, der Verwaltung und Vorbereitung von Daten und weitere $20 \%$ damit, Arbeiten aufgrund von fehlenden Informationen doppelt zu erledigen [2].

Einer der Hauptgründe dafür ist, dass Daten oft parallel existieren ohne jegliche Verbindung zueinander. Die unterschiedlichen Fachabteilungen sind nicht miteinander vernetzt oder Mitarbeiter behalten ihr Wissen für sich (Information Hoarding), um sich vermeintlich unentbehrlich zu machen. Hinzu kommen unterschiedliche Wissensquellen, die nicht miteinander verbunden sind: E-Mail-Postfächer, Ablagesysteme, Archive etc.

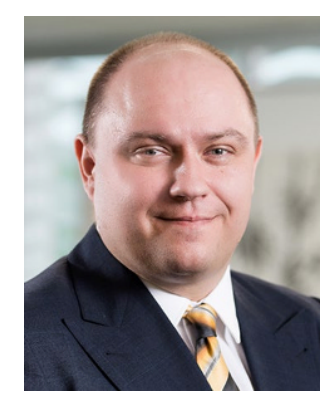

Daniel Fallmann $(\square)$ Gründer \& CEO Mindbreeze GmbH office@mindbreeze.com

${ }^{1}$ Linz, Österreich

Ein Mehrwert lässt sich aber nur dann generieren, wenn die vorhandenen Daten Mitarbeitern im richtigen Moment und Kontext zur Verfügung stehen - als Entscheidungsgrundlage oder um Workflows und Arbeitsprozesse effizienter zu gestalten.

\section{Informationen verstehen und ganzheitlich extrahieren}

Insight Engines nutzen Methoden der künstlichen Intelligenz wie Machine und Deep Learning sowie Formen der Spracherkennung und kombinieren sie mit bekannten Möglichkeiten von Enterprise-Search-Lösungen, um die Daten aus den unterschiedlichen Datenquellen zu verknüpfen. Das Ergebnis: Komplexe Zusammenhänge und Sachverhalte stehen den Anwendern auf einen Blick (Holistic View) klar ersichtlich zur Verfügung.

Bei der Verarbeitung sämtlicher Daten aus den an die Insight Engine angebundenen Fachanwendungen oder Datenbanken (Cloud, Archive, Netzwerke etc.) werden die Inhalte semantisch analysiert und bestehende Zusammenhänge er-

Wirtschaftsinformatik \& Management 2021 • 13 (3): 247-248

https://doi.org/10.1365/s35764-021-00320-3

Angenommen: 20. Januar 2021

Online publiziert: 15. Februar 2021

(c) Springer Fachmedien Wiesbaden GmbH, ein Teil von Springer Nature 2021 
kannt. Dadurch können Fragen von Anwendern mit den extrahierten Fakten punktgenau beantwortet werden. Da sich die Art und Weise nach Informationen zu suchen, in den letzten Jahren stark verändert hat - Suchanfragen bestehen heute nicht mehr aus reinen Schlagworten, sondern aus echten Fragestellungen (Conversational Search) -, ist eine umfassende KI erforderlich, um die Suchanfragen verstehen und verarbeiten zu können.

Die linguistische Interpretation erfolgt dabei durch Natural Language Processing (NLP). Hier handelt es sich um eine Methode, die menschliche, natürliche Sprache mithilfe von Regeln und Algorithmen computerbasiert verarbeitet. So versteht die Insight Engine die bestehenden Inhalte und transformiert sie in Informationen. Das gilt sowohl für einzelne Wörter und Sätze als auch für Zusammenhänge, Texte und Sachverhalte.

Basierend auf Natural Language Understanding (NLU) ermittelt und interpretiert die Insight Engine die Absicht des Anwenders. Damit geht NLU über das reine Verstehen von Wörtern und Sätzen hinaus. Im Fokus steht hier die detaillierte Interpretation der Bedeutung eines Satzes, einer Anfrage oder einer Aussage (Behavior Intent). Trotz menschlicher Fehler (Interpunktion-, Rechtschreib-, Grammatik- oder Tippfehler) ist eine Insight Engine damit in der Lage, auf Fragestellungen mit „wie“, „wo“, „Wer“, „warum“, „wann“ etc. adäquat zu reagieren und explizite Antworten zu geben.

Im nächsten Schritt extrahiert die Insight Engine die der Anfrage entsprechenden Informationen, bereitet sie dem Anwender auf und stellt zuvor sämtliche Zugriffsberechtigungen sicher.

\section{Kontextsensitive Sichten}

So erhält der Kundenservice eine andere Sicht auf die gesuchten Themen als die Geschäftsführung. Dabei werden nicht nur die Rolle des Benutzers und das Organigramm berücksichtigt, sondern es fließen auch Kontextinformation wie der jeweilige Arbeitsschritt, die Lokation, die Zeit, das bisherige Verhalten und Interesse und vieles weitere mit ein. Damit lässt sich zum einen sicherstellen, dass nur Mitarbeiter mit den entsprechenden Berechtigungen Zugang zu kritischen Informationen erhalten, zum anderen bekommt jeder genau die Informationen angezeigt, die benötigt werden.

Künstliche Intelligenz, besser gesagt trainierte neuronale Modelle arbeiten im Hintergrund und sorgen dafür, dass sich die Insight Engine kontinuierlich durch die Analyse von beispielsweise Suchabfragen, neuer Datensätze oder des Nutzer-

\section{Über Mindbreeze}

Die Mindbreeze $\mathrm{GmbH}$ ist ein führender Anbieter von Appliances und Cloud-Services für Information Insight, angewandte künstliche Intelligenz und Wissensmanagement. Die Produkte ermöglichen eine konsolidierte Sicht auf das Unternehmenswissen unabhängig davon, wo und wie dieses gespeichert ist. www.mindbreeze.com

verhaltens weiterentwickelt. Basierend auf den Interaktionen mit den Ergebnissen errechnen Relevanzmodelle die Wichtigkeit für den Anwender und personalisieren die Darstellungsweisen.

In einer hochfrequenten Zeit, wo in Millisekunden große Datenmengen produziert werden, ist der klassische Ansatz für Wissensmanagement, Informationen aus den unterschiedlichsten Quellen in eine zentrale Datenbank zu übertragen, um Wissen zentral zur Verfügung zu stellen, nicht mehr zeitgemäß. Heute muss der einfache und schnelle Zugriff auf Informationen im Vordergrund stehen, unabhängig davon, wo diese gespeichert sind. Der Produktivitätssprung, der mit dem Einsatz von Insight Engines einhergeht, verleiht Unternehmen einen Innovationsschub und generiert auf diese Weise einen nachhaltigen Wettbewerbsvorsprung.

\section{Literatur}

[1] Gartner Inc. (2020). 9 Future of Work Trends Post-COVID-19. https://www.gartner.com/smarterwithgartner/9-future-of-worktrends-post-covid-19/. Zugegriffen: 30. Nov. 2020.

[2] Curry, D. (2018). IDC: Data Professionals Unproductive for $50 \%$ of Working Day. https://www.rtinsights.com/data-professionals-unproductive/. Zugegriffen: 1. Dez. 2020.

Springer Professional $\mid \begin{aligned} & \text { Mehr zum Thema finden Sie online } \\ & \text { www.springerprofessional.de/wum }\end{aligned}$ 